\title{
An innovative training for nurses in sexual and reproductive health
}

\author{
Shelley Mehigan, ${ }^{1}$ Janice Burnett ${ }^{2}$
}

\author{
${ }^{1}$ Nurse Specialist in \\ Contraception, Bournemouth, \\ UK \\ ${ }^{2}$ Nurse Consultant in Sexual \\ Health, Sexual Health Service, \\ Royal Berkshire NHS Foundation \\ Trust, Reading, UK
}

\section{Correspondence to} Ms Shelley Mehigan; shellraine@aol.com

Received 16 April 2012 Accepted 8 May 2012
Why was a new approach needed?

There is no longer a nationally recognised, standardised training for nurses in sexual and reproductive health $(\mathrm{SRH})^{1}$ but increasingly services and general practices are using the skills of nurses to deliver contraception and sexual health in clinical practice. In order to provide nurses with the competencies and skills to deliver optimum care at a recognised high standard, and having successfully piloted the use of the, then, new Diploma of the Faculty of Sexual and Reproductive Healthcare (DFSRH) components into the previous University of Reading course, ${ }^{1}$ the Nurse Leads in Berkshire decided to develop a training programme for nurses that matches that undertaken by doctors for the DFSRH qualification. The DFSRH is currently being independently reviewed by Professor Ed Peile of the University of Warwick and the Reading team and nurses fed their experiences into this review.

\section{How does the new training work?}

Nurses are identified either by direct contact with the services in East and West Berkshire or via the local nurse training commissioners. The two Nurse Leads support these nurses to access and complete all the elements of the DFSRH. After completing the e-SRH e-learning theory element, nurses attend a Course of 5 (C5) either individually, in small groups or as part of a C5 for doctors depending on timing and availability. Clinical experience and assessment is arranged in local clinics and practices with experienced nurse trainers and Faculty training doctors using the Assessment of Clinical Practice (ACP) tools developed by the Faculty.

Throughout the training nurses use a logbook (adapted from that used previously by the FSRH for doctors) to record clinical experience and reflections of practice as they are not currently able to access the e-Portfolio available to doctors. On completion nurses are given a Certificate of Completion of Clinical Practice, which they are advised to keep, together with the certificates from the e-learning sessions and C5, and their logbook as part of their portfolio of evidence of competence to practice.

\section{Does it work?}

The 13 nurses who have so far completed this training (no drop-outs or failures) have evaluated it well. The response from trainers (doctors and nurses) has been positive, particularly with regard to the level of knowledge that the trainees have when they embark on clinical assessment. The Lead Nurses believe that this course is 'fit for purpose', accessible, flexible and cost effective and could be replicated in other areas where no alternative exists. In addition it could be used flexibly by those currently offering SRH training in other settings. The Margaret Pyke Centre has used a similar format to train nurses who have been employed specifically to undergo the training $^{1}$ and the Berkshire model has since been adopted by Oxfordshire CASH services.

\section{What are the benefits of this method of training?}

- Standardising training across the UK: recognisable competences are more transferable.

- Matched, multidisciplinary skills: allows increased flexibility in service delivery. A basis from which nurses could go on to do further training to fit implants and intrauterine devices (IUDs). As they are increasingly contributing to the training of doctors it makes sense that they have the same SRH training.

- Cost-effective: the theory component is free and can be accessed to suit individual trainees, at work or home. Less time out of practice to attend study days.

- Flexible: as well as the theory, C5 can be done flexibly. With standardised elements, trainees could move more easily from one area to another to complete.

- Accessible: particularly appropriate for nurses who do not need or do not 
wish to do an academic course (e.g. practice nurses or those who already have a first and/or higher degree). Without the need for academic input the training could be done anywhere that has access to clinical practice for assessment.

\section{What are the challenges?}

The main issue for some is around accreditation with the belief that this can only be achieved through academic institutions or organisations like the Royal College of Nursing (RCN). From the competence to practice aspect this is not the case and does not, in any case, guarantee standardisation apart from an academic standpoint. Nurses need to be able to demonstrate their competence to practise in order to comply with the Nursing and Midwifery Council Code. ${ }^{2}$ However universities and higher education institutions (HEIs) could incorporate the core elements into any of the post-registration and higher degree courses they offer that include SRH, which would also ensure standardisation nationally. There is little value in having one university accredit this training as it would not be easily transferable to other areas nor for the RCN to accredit it as this would incur additional costs which will vary for members and non-members. Perhaps one alternative suggestion could be for the Deaneries to support and 'badge' training similar to the Berkshire one.

\section{The future}

We still firmly believe that the best way forward for nurse training in SRH would be for the FSRH to be able to accredit nurses or to open up the Diploma to them. As well as ensuring a competent, matched, multidisciplinary workforce, the Faculty already provides the post-qualification support and updating necessary to all clinicians working in this field. Nurses would also have the same foundation on which to build further training, such as fitting and removal of subdermal implants and IUDs and teaching qualifications. Ideally these would also be the versions offered by the Faculty to doctors, which would also make it easier for them to recognise nurses as trainers. This view is held by many nurses and doctors in the field (as demonstrated by the 17 letters published in this Journal in response to the Personal View article by Mehigan et al. ${ }^{1}$ in 2010) and we sincerely hope that some mechanism can be found to achieve this.

If this is not possible then we would like to propose that other services, practices, individuals and HEIs use the excellent training that is already out there rather than reinventing lots of different 'wheels', which will inevitably lead to a 'wobbly', mismatched workforce at a time when quality, consistency and standardised excellence in practice should be our collective aim.

Competing interests None.

Provenance and peer review Not commissioned; externally peer reviewed.

\section{References}

1 Mehigan S, Moore W, Hayes L. Nurse training in sexual and reproductive health. J Fam Plann Reprod Health Care 2010;36:5-6.

2 Nursing and Midwifery Council. The Code: Standards of Conduct, Performance and Ethics for Nurses and Midwives. 2008. http://www.nmc-uk.org/Nurses-and-midwives/The-code/ [accessed 10 April 2012]. 